\title{
Detailed structural and mechanical response of wet foam to the settling particle
}

\section{Supporting Information}

Zefeng Jing, Shuzhong Wang and Zhiguo Wang

Key Laboratory of Thermo-Fluid Science and Engineering of MOE, School of Energy and Power

Engineering, Xi'an Jiaotong University, Xi'an, Shanxi, China

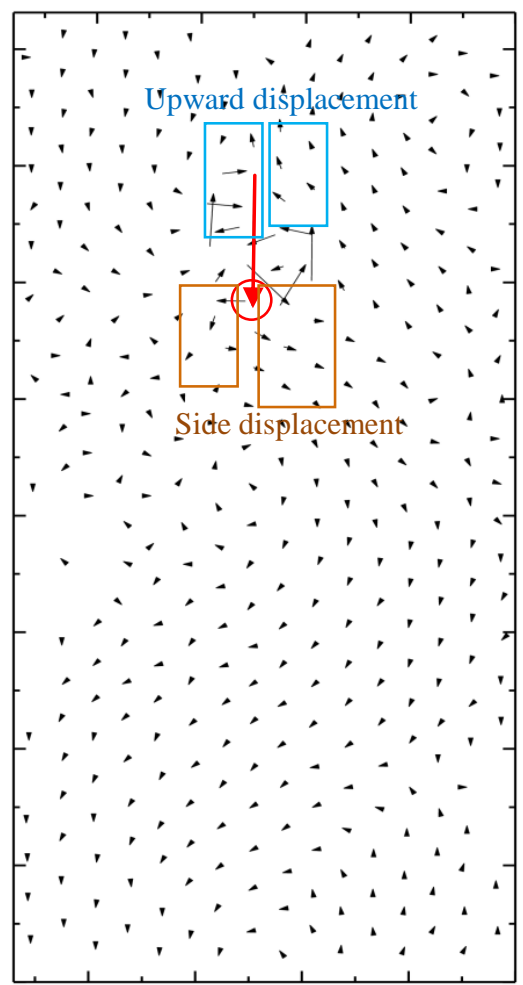

(a) 300-600 iterations

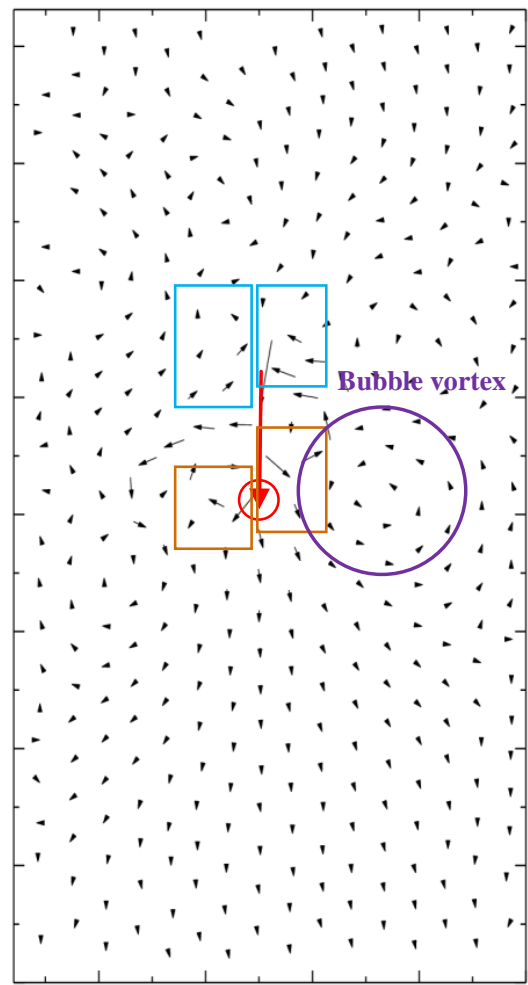

(b) 800-1100 iterations

Figure S-1. Displacement fields of bubbles in different iteration intervals during the sedimentation. The red arrows denote the displacement of the particle during these intervals. There is also bubble vortex at the side of the settling particle.

In order to analyze changes in the thickness of liquid lamellae separating the bubbles in the front and wake of the falling particle conveniently, displacement fields of bubbles and partial bubbles around the particle are shown in Figures S-1 and S-2. From the displacement fields in 
Figure S-1, we can find that some bubbles in the wake of the particle have upward displacements and tend to move towards the middle part which the particle passes through. This will make these bubbles gradually come together. Accordingly, it is obviously seen in Figure S-2 that the thickness of liquid lamellae between the two bubbles in the wake decreases gradually.

In addition, according to Figures $\mathrm{S}-1$ and $\mathrm{S}-2$, it can also be seen that the bubbles in front of the particle are squeezed to both sides of the particle as the particle settles in the wet foam. Thus, these bubbles have side displacements, which leads to the detachment of conterminal bubbles (T1 event). This obviously causes the gradual increase of lamellae thickness until the liquid lamellae disappears (see Figure S-2).

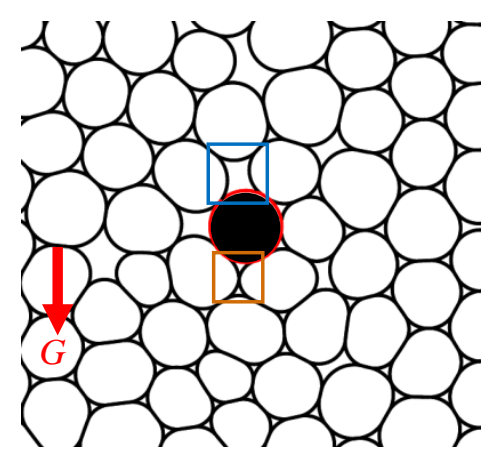

(a) The $1405^{\text {th }}$ iteration

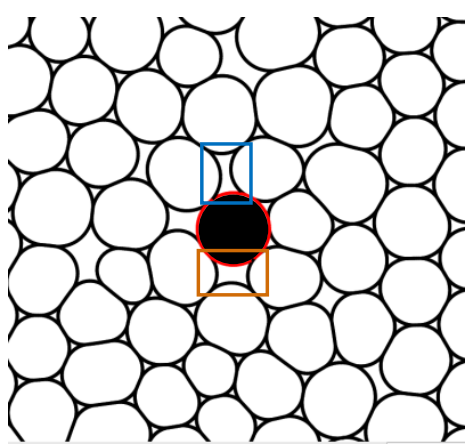

(b) The $1410^{\text {th }}$ iteration

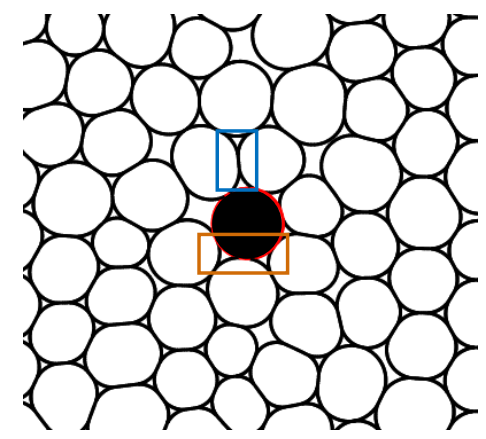

(c) The $1415^{\text {th }}$ iteration

Figure S-2 Evolution of the bubbles around the particle during the sedimentation. The red arrow denotes the direction of gravity. 